\title{
What is the optimal timing for Thoracic Endovascular Aortic Repair in uncomplicated Type B aortic dissection? Facts, fallacies and the future
}

Matti Jubouri ${ }^{1}$, Mohamad Bashir ${ }^{2}$, Sven Zhen Cian Patrick Tan ${ }^{3}$, Damian Bailey ${ }^{4}$, Richard Anderson $^{5}$, CHRISTOPH NIENABER ${ }^{6}$, JOSEPH Coselli ${ }^{7}$, and Iam Williams ${ }^{5}$

${ }^{1}$ Hull York Medical School

${ }^{2}$ Velindre University NHS Trust

${ }^{3}$ Queen Mary University of London Barts and The London School of Medicine and Dentistry

${ }^{4}$ University of South Wales Faculty of Life Sciences and Education

${ }^{5}$ University Hospital of Wales

${ }^{6}$ Royal Brompton and Harefield NHS Foundation Trust

${ }^{7}$ Baylor College of Medicine Michael E DeBakey Department of Surgery

November 10, 2021

\begin{abstract}
Background: Uncomplicated Stanford Type B aortic dissection (un-TBAD) is characterised by a tear in the aorta distal to the left subclavian artery without ascending aorta and arch involvement. Optimised cardiovascular control (blood pressure and heart rate) is the current gold standard treatment according to current international guidelines. However, emerging evidence indicates that Thoracic Endovascular Aortic Repair (TEVAR) is both safe and effective in the treatment of un-TBAD with improved long-term survival outcomes in combination with optimal medical therapy (OMT) relative to OMT alone. However, the optimal timeframe for intervention is not entirely clarified. Aims: This review critically addresses current state-of-the-art comparing TEVAR with OMT and corresponding clinical outcomes for un-TBAD based on timing of intervention. Methods: We carried out a comprehensive literature search on multiple electronic databases including PUBMED and Scopus in order to collate all research evidence on timing of TEVAR in uncomplicated Type B aortic dissection. Results: TEVAR has proven to be a safe and effective treatment for un-TBAD in combination with OMT through comparable survival outcomes, improved aortic remodelling, and relatively low periprocedural added risks. Though the timing of intervention remains controversial, it is becoming clear that performing TEVAR during the subacute phase of un-TBAD yields better outcomes compared to earlier and delayed (>90 days) intervention. Conclusions: Further research is required into both short and long-term outcomes of TEVAR in addition to its optimal therapeutic window for un-TBAD. With stronger evidence, TEVAR is likely to be adopted as the gold-standard intervention for un-TBAD with definitive timeframe guidelines.
\end{abstract}

What is the optimal timing for Thoracic Endovascular Aortic Repair in uncomplicated Type B aortic dissection?

Facts, fallacies and the future

Matti Jubouri ${ }^{1}$, Mohamad Bashir MD PhD MRCS ${ }^{2}$, Sven ZCP Tan ${ }^{3}$, Damian Bailey FRSC FPVRI FACSM FTPS $^{4}$, Richard Anderson BSc MBBS MD ${ }^{5}$, Christoph A. Nienaber MD PhD MRCP ${ }^{6}$, Joseph S. Coselli $\mathrm{MD}^{7}$, Ian Williams MD FRCS ${ }^{8}$ 
1. Hull York Medical School, University of York, York, UK

2. Velindre University NHS Trust, Health Education \& Improvement Wales, Vascular \& Endovascular Surgery, Wales, UK

3. Barts and The London School of Medicine and Dentistry, Queen Mary University of London, UK

4. Neurovascular Research Laboratory, Faculty of Life Sciences and Education, University of South Wales, Pontypridd, UK

5. Department of Cardiology, University Hospital of Wales, Heath Park, Cardiff, UK

6. Cardiology and Aortic Centre, Royal Brompton and Harefield Hospital NHS Foundation Trust, London, UK.

7. Division of Cardiothoracic Surgery, Michael E. DeBakey Department of Surgery, Baylor College of Medicine, Houston, Tex; Department of Cardiovascular Surgery, Texas Heart Institute, Houston, Tex; CHI St Luke's-Baylor St. Luke's Medical Center, Houston, Tex.

8. Department of Vascular Surgery, University Hospital of Wales, Heath Park, Cardiff, UK

Correspondence:

Ian Williams MD FRCS

Department of Vascular Surgery

University Hospital of Wales Heath Park

Cardiff

UK

Email: Ian.Williams5@Wales.nhs.uk

Keywords: Type B Aortic Dissection, Aortic Dissection, Aneurysm, Aortic Surgery

COI: None

Funding: Damian Bailey is funded by a Royal Society Wolfson Research Fellowship (\#WM170007) and separate grants from the Royal Society (IES/R2/192137) and Japan Society for the Promotion of Science (JSPS/OF317). Otherwise no funding received.

Ethical approval: No ethical approval required for this review.

Guarantor: Ian Williams (IW)

Contributorship: MJ, MB, and SZCPT involved in literature review design and writing. MB, DMB, RA, $\mathrm{CN}$, JC and IW involved in manuscript revision.

Acknowledgements: None

Abstract

Background: Uncomplicated Stanford Type B aortic dissection (un-TBAD) is characterised by a tear in the aorta distal to the left subclavian artery without ascending aorta and arch involvement. Optimised cardiovascular control (blood pressure and heart rate) is the current gold standard treatment according to current international guidelines. However, emerging evidence indicates that Thoracic Endovascular Aortic Repair (TEVAR) is both safe and effective in the treatment of un-TBAD with improved long-term survival outcomes in combination with optimal medical therapy (OMT) relative to OMT alone. However, the optimal timeframe for intervention is not entirely clarified.

Aims: This review critically addresses current state-of-the-art comparing TEVAR with OMT and corresponding clinical outcomes for un-TBAD based on timing of intervention.

Methods: We carried out a comprehensive literature search on multiple electronic databases including PUBMED and Scopus in order to collate all research evidence on timing of TEVAR in uncomplicated Type 
B aortic dissection.

Results: TEVAR has proven to be a safe and effective treatment for un-TBAD in combination with OMT through comparable survival outcomes, improved aortic remodelling, and relatively low periprocedural added risks. Though the timing of intervention remains controversial, it is becoming clear that performing TEVAR during the subacute phase of un-TBAD yields better outcomes compared to earlier and delayed ( $>90$ days) intervention.

Conclusions: Further research is required into both short and long-term outcomes of TEVAR in addition to its optimal therapeutic window for un-TBAD. With stronger evidence, TEVAR is likely to be adopted as the gold-standard intervention for un-TBAD with definitive timeframe guidelines.

\section{Introduction}

\section{Aortic Dissection}

Aortic dissection (AD) is a subclass of acute aortic syndromes encompassing life-threatening aortic pathologies that also include penetrating atherosclerotic aortic ulcer and intramural haematoma [1]. The pathology of dissection involves a tear in the aortic intima where blood circulates from the true lumen (TL) through the entry tear to the false lumen (FL) that expands over time, which could lead to either compression of the TL and/or to FL rupture. The tear can also propagate antegrade (more common) and/or retrograde due to the pulsating pressure head. This expansion of the FL and propagation of the original entry tear may result in malperfusion and end-organ ischaemia (malperfusion syndrome) [2-4]. AD can happen at relatively normal aortic diameter but the aortic haematoma is likely to expand following the inception of dissection, with growth rates varying depending on dissection zone [5].

AD affects an estimated 3-4 people per 100,000 annually, most of whom are men in their 60s with a history of hypertension and/or diseased blood vessels. Other predisposing factors leading to AD include genetic connective tissue disorders such as Marfan and Ehlers-Danlos syndrome, hereditary vascular disease including Loeys-Dietz syndrome, vascular inflammation, and trauma [2][3][6]. AD can be further classified based on anatomy using two universal systems, DeBakey and Stanford. The DeBakey system is based on the location of the original entry tear and extent of the dissection [2][7]. Daily and colleagues [8] at Stanford University developed the Stanford system and categorised AD into type A and type B depending on whether the ascending aorta is involved in the tear [2][9]. The Society of Thoracic Surgery (STS)/Society of Vascular Surgery (SVS) released a more recent and improved classification system that provides a more structured and systematic approach to classifying all types of AD. The reporting standards are similar to those of the Stanford system with some amendments such as the inclusion of dissection tears that originate within the arch itself (Figure 1) [10][11]. The current review will focus specifically on uncomplicated Stanford Type B $\mathrm{AD}$ (un-TBAD).

\section{Stanford Type B Aortic Dissection}

Stanford Type B AD (TBAD) involves a tear in the aorta distal to the left subclavian artery (LSA) without any involvement of the ascending aorta or arch proximal to the LSA (Figure 2). It is equivalent to a DeBakey III (IIIa and IIIb) dissection [10]. As illustrated in Figure 1. The STS/SVS system defines TBAD as a dissection with an aortic entry tear originating in zone 1 or beyond (zones 2-11) [10][11]. TBAD can be further categorised by time of onset and presence of complications [2][12]. Different studies report different time classification of TBAD, however, it is generally accepted as being acute or chronic. Acute TBAD presents within 14 days of symptom onset while chronic TBAD is diagnosed 14 days following symptoms. A subacute phase has been described in some studies as a duration of 15-90 days following symptoms [2][3][12]. The International Registry of Acute Aortic Dissection divides AD based on time into hyperacute (symptom onset up to 24 hours), acute (2-7 days), subacute (8-30 days), and chronic ( $>30$ days) [9][13]. The dissecting membrane in chronic dissections becomes less compliant, which appears as a straight line on computed tomography (CT) (Figure 3), while the more compliant septum in acute dissection present as a wavy line on CT imaging. 
In terms of complications, complicated TBAD (co-TBAD) is associated with aortic rupture, hypotension/shock, malperfusion, neurological signs, acute renal failure, recurrent or refractory pain, refractory hypertension, and/or early aortic dilatation or progression of disease [2][12]. On the other hand, uncomplicated TBAD (un-TBAD) comprises about $75 \%$ of TBADs and does not present with the above complications. Since the introduction of thoracic endovascular aortic repair (TEVAR) for TBAD it has become the gold standard intervention for co-TBAD. Yet, un-TBAD is still treated conventionally with optimal medical therapy (OMT), with the exception of acute high-risk cases who are directly offered TEVAR. It is also important to realise that $20-50 \%$ of un-TBADs will progress to become complicated and eventually require some intervention [2][9][14]. Historically, un-TBAD was repaired with open surgery once a diameter-based threshold of repair, set and challenged as at 5.5 or $6.0 \mathrm{~cm}$, was attained or complications developed [15-17]. However, TEVAR is gaining increasing popularity as a treatment option for un-TBAD due to its improved long-term outcomes in combination with best medical therapy [2][3][18][19]. However, the optimal timeframe for intervention remains to be established.

\section{TEVAR for un-TBAD: the dilemma of 'why?' and 'when?'}

While research is suggesting that endovascular repair of TBAD could be successfully performed in the subacute and early chronic phases, there remains concerns about the ability of TEVAR to remodel the aorta (FL thrombosis, increase in TL dimeter, and decrease in FL diameter) due to the fact that the dividing septum becomes less compliant over time, particularly when the chronic phase is reached [15]. This led to a paradigm shift to assess if performing TEVAR well below the traditional threshold of intervention in early phase of an un-TBAD would have greater success in remodelling the distal aorta. Repair in the acute phase must weigh any purported remodelling benefits against added risks in the acute phase such as retrograde type A dissection or rupture. Perhaps there is a point along the continuum between acute un-TBAD at a relatively small aortic diameter to chronic un-TBAD at $5.5 \mathrm{~cm}$ that would optimise the benefits of TEVAR and mitigate its risks.

\section{Management of Uncomplicated Type B Aortic Dissection}

OMT also known as best medical therapy (BMT), has traditionally been the first line treatment for un-TBAD irrespective of any additional intervention. Open surgery is associated with increased morbidity/mortality and subsequently abandoned [1][9]. The ultimate goal of OMT according to the American College of Cardiology Foundation (ACCF) involves pharmacological control of BP (systolic BP $<100-120 \mathrm{mmHg}$ ) and HR $(<60 \mathrm{~b} / \mathrm{min})$ to minimise shear forces and circumferential strain on the aortic wall and avoid FL pressurisation [12][20]. Pharmacotherapy includes selective beta-blockers (first-line), diuretics, calcium channel blockers, angiotensin-converting-enzyme (ACE) inhibitors, and alpha blockers (less common) [21]. There is evidence conservative treatment for un-TBAD has a 10\% (30-day) mortality and up to $25 \%$ requiring intervention within 4 years principally due to aortic degeneration [22]. While TEVAR is the recommended treatment choice as per international guidelines for co-TBAD, it is still being evaluated for un-TBAD.

Several studies, such as STABLE and STABLE II by Lombardi et al., proved that TEVAR is highly effective in co-TBAD due to its promotion of favourable aortic remodelling, false lumen thrombosis, and overall survival relative to open surgery [23][24]. The INSTEAD, INSTEAD-XL, and ADSORB randomized control trials investigated the use of OMT alone for un-TBAD versus the combination of OMT with TEVAR, and reported on several early and late outcomes. To what extent does TEVAR offer un-TBAD patients with comparable improvements in clinical outcomes as those with co-TBAD [22][25-27]?

As mentioned previously, 20-50\% of un-TBADs will progress to become complicated and eventually require complex intervention. Although un-TBAD may not be symptomatic in the chronic phase but it sometimes possesses a high risk of progression and development of complications due to certain physiological and radiological conditions. There high risk-indicating parameters include total aortic diameter $>44 \mathrm{~mm}$, FL diameter $>22 \mathrm{~mm}$, a patent or partial thrombosed FL, primary entry tear diameter $>10 \mathrm{~mm}$, and age of $>60$. It is vital to identify these cases early on and intervene accordingly to prevent disease progression [3] [28] [29]. 


\subsection{INSTEAD}

The INSTEAD (The INvestigation of STEnt Grafts in Aortic Dissection) trial included un-TBAD patients in the subacute and chronic phases. One hundred and forty patients were randomly allocated to two treatment groups. The first group subjected to elective stent-graft placement in addition to OMT $(\mathrm{n}=72)$; the second group was assigned to OMT alone $(n=68)$ with BP monitoring. The primary outcome was all-cause mortality at two years. Secondary outcomes included aorta-related deaths (rupture), disease progression(with conversion or additional endovascular or open surgery), and aortic remodelling (FL thrombosis as well as TL and FL diameters). Data over a two-year follow-up period showed no difference between the two groups in all-cause mortality $(95.6 \pm 2.5 \%$ with OMT versus $88.9 \pm 3.7 \%$ with TEVAR, $\mathrm{P}=0.15)$, aorta-related death $(97.0 \pm 2.0 \%$ with OMT versus $94.4 \pm 2.7 \%$ with TEVAR, $\mathrm{P}=0.44)$. However, three neurological complications occurred in the TEVAR group versus only one with medical treatment (30-day, not significant). In addition, there was no difference in incidence of persistent paraplegia/paraparesis and major stroke within two years of randomization ( $\mathrm{P}=0.90$ and $\mathrm{P}=0.53$, respectively) More importantly, TEVAR patients showed improved aortic remodelling compared to the OMT group (91.3\% and $19.4 \%$, respectively, $\mathrm{P}<0.001)$. Collectively, these findings highlighted that despite improved aortic remodelling, TEVAR failed to incur a morbidity and mortality benefit [25].

\subsection{INSTEAD-XL}

Nienaber et al. extended the follow-up up period of the INSTEAD RCT to five years. TEVAR was shown to reduce all-cause mortality $(11.1 \%$ versus $19.3 \% ; \mathrm{P}=0.13)$, aorta related mortality $(6.9 \%$ versus $19.3 \%$; $\mathrm{P}=0.04)$, and progression of dissection (27.0\% versus $46.1 \% ; \mathrm{P}=0.04)$ relative to OMT alone. Complete stent graft-induced FL thrombosis was observed in $90.6 \%$ of elective TEVAR cases at five years relative to $22.0 \%$ with OMT only $(\mathrm{P}<0.0001)$. TEVAR was necessary in fourteen cases randomized to OMT alone and seven additional stent grafts were required in the TEVAR group. Furthermore, three TEVAR patients underwent open surgical conversion as well as four cases in the OMT alone group. Events leading to crossover or open conversion occurred more frequently with OMT than after TEVAR. Overall, TEVAR conferred a long-term survival advantage with the Kaplan-Meier curves demonstrating survival benefit (all-cause and aorta-specific mortality) with TEVAR seen between 2 and 5 years $(\mathrm{P}=0.0003$ and $\mathrm{P}=0.0005$, respectively). The authors recommended considering pre-emptive TEVAR in stable patients with suitable anatomy to improve late outcomes [26].

\subsection{ADSORB}

The prospective multicentre "Acute Dissection: Stent graft OR Best medical therapy" (ADSORB) trial randomised 61 patients with acute un-TBAD into two groups to compare BMT $(n=31)$ with BMT and stent grafting of the primary entry tear $(n=30)$. Patients were followed up at set intervals after acute dissection. Primary outcomes included thrombosis of the FL and aortic enlargement at one year as well as aortic rupture. No deaths occurred in either group during the first 30 days, still, there were three crossovers from the BMT to the BMT+TAG group due to progression of disease within 1 week. Brunkwall et al. reported results after one year of surveillance. FL thrombosis did not occur in $97 \%$ of patients treated with BMT, conversely, FL thrombosis was observed in $57 \%$ of the BMT+TAG group $(\mathrm{P}<0.001)$. The average maximum FL and TL diameters for TAG+BMT were $18.5 \mathrm{~mm}$ and $32.2 \mathrm{~mm}$, respectively, versus $25.1 \mathrm{~mm}$ and 25.5 $\mathrm{mm}$, respectively, for BMT $(\mathrm{P}<0.001)$. A maximum FL diameter decrease of $7.0 \mathrm{~mm}$ was recorded in the BMT+TAG group compared to an increase of $4.3 \mathrm{~mm}$ in the BMT group $(\mathrm{P}<0.001)$. In addition, no significant difference was observed in aortic dilation $(\mathrm{P}=0.5)$. Finally, it was concluded that the Gore TAG device can be safely used to treat un-TBAD [22][27].

\subsection{Observational studies}

Several observational studies compared the two treatment options and reported favourable outcomes for TEVAR for un-TBAD. For example, Qin et al. [30] presented a multicentre retrospective study of 338 un-TABD patients (184 TEVAR and 154 BMT) that ruled in favour of TEVAR. Early events and 30day mortality were similar between groups while aortic-related adverse events and all-cause mortality were 
lower with TEVAR ( $\mathrm{P}=0.025$ and $\mathrm{P}=0.01$, respectively) relative to BMT. Conversely, Xiang et al. [31] retrospectively analysed data from 357 patients with acute un-TBAD (191 TEVAR and 166 BMT) and indicated that TEVAR was associated with more early complications e.g. acute renal failure, type I endoleak, stroke, rupture, retrograde Type A dissection (RTAD), and organ failure (events occurring in-hospital or within 30 days after diagnosis), but similar 30-day mortality. TEVAR however still improved mid-term survival and decreased risk of late death as well as late aortic ruptures (occurring

$>30$ days after diagnosis). Freedom from all-cause mortality and aortic-related death were significantly higher with TEVAR; thus, the authors recommended it as first-line treatment for acute un-TBAD [31].

In a meta-analysis, Hossack et al. [18] reported a lower risk of all-cause and aorta-related mortality with TEVAR than BMT $(\mathrm{P}<0.001$ and $\mathrm{P}=0.001$, respectively $)$ for acute and subacute un-TABD. Nevertheless, TEVAR was associated with significantly higher risk of early stroke $(\mathrm{P}=0.002)$, but similar inpatient mortality and reintervention rates to BMT. Another meta-analysis by Enezate et al. [19] reported comparable rates of short, intermediate, or mid-term all-cause mortality and aortic dilation between TEVAR and medical therapy in acute dissection. TEVAR yet showed significantly lower 1-year risk of aortic rupture $(\mathrm{P}=0.01)$. Chen et al. [32] demonstrated that TEVAR performed in the acute stage of dissection was associated with an increased risk of RTAD.

The prevailing consensus indicates that TEVAR provides comparable benefit to current medical therapies for un-TBAD. Additional research is warranted to determine the potential early, mid-term, and late outcome benefits of TEVAR in un-TBAD to further optimise patient management. A summary of all the above findings can be found in Table 1.

Timing of TEVAR: When to intervene?

Timing of TEVAR for all TBAD has provoked considerable discussion over the last twenty years. However for un-TBAD this does remain a controversial issue as there is always those who suggest TEVAR is unnecessary and optimum treatment in medical therapy alone. For those presenting with a complicated TBAD, treatment by TEVAR is more easily defended. This is due to risk of adverse events both short and long term if intervention by TEVAR is not performed. Intervention in the hyperacute phase by TEVAR is invariably in a high risk situation, where if nothing is done then a significant mortality rate might be expected due to a distinct lack of research. Data on TEVAR timing is varied, herein we seek to analyse and evaluate the current available evidence. These findings are summarised in Table 2.

Most of the data concerning the timing of TEVAR is extracted from studies concerning co-TBAD, while the fate of un-TBADs remains to be established. A retrospective analysis by Lee et al. [33] split 87 patients into acute/subacute ([?]6 weeks, $\mathrm{n}=35$ ), early chronic ( 6 weeks to 1 year, $\mathrm{n}=20$ ), and late chronic $(>1$ year, $\mathrm{n}=32$ ) groups based on timing of TEVAR after symptom onset of the TBAD. Clinical outcomes including aortic remodelling (maximum total aorta diameter and TL/FL diameters) were similar in the acute/subacute and early chronic phases of TBAD, but superior to those in the late chronic TBAD group. Five-year overall mortality and aorta-related mortality were slightly lower in the acute/subacute and early chronic groups compared to late chronic ( $2.9 \%$ versus 0 versus $12.5 \%$, respectively, $\mathrm{P}=0.108$, and $2.9 \%$ versus 0 versus $9.4 \%$, respectively). Desai et al. [34] analysed data from 132 TEVARs out of which 70 were performed within 48 hours of presentation (Acute-Early), 44 performed between 48 hours and 14 days from presentation (Acute-Delayed), and 18 performed between 14 days and 6 weeks of presentation (Subacute). The results demonstrated that earlier intervention with TEVAR was associated with increased periprocedural complications but comparable late outcomes.

The VIRTUE Registry, a prospective, non-randomised multicentre European Clinical Registry focused on 100 patients with acute $(n=50)$, subacute $(n=24)$, and chronic co-TBAD $(n=26)$. Thirty-day mortality and stroke rates were $8 \%, 0 \%$, and $0 \%$ for acute, subacute, and chronic lesions, respectively. Three-year followup results showed no difference for all-cause mortality $(\mathrm{P}=0.20)$, dissection-related mortality $(\mathrm{P}=0.48)$ and overall aortic reintervention $(\mathrm{P}=0.19)$ between groups. They observed in acute co-TABD one stroke, one rupture and two RTAD. Notably, no cases of RTAD in the subacute and chronic groups were noted. However, 
one with a chronic TBAD suffered a rupture on follow up. With regards to FL volume change, this was higher in the acute than in the chronic group $(\mathrm{P}<0.001)$ and similar when the subacute and chronic group were compared $(\mathrm{P}=0.004)$. However, there was no difference in FL area change between the acute and subacute groups. As a conclusion, the authors stated that the retention of aortic plasticity in the subacute group extends the therapeutic window for TEVAR to 90 days [35][36].

A single centre study by Xie et al. [37] retrospectively examined 267 patients with un-TBAD, carrying risk factors for complications or dissection progression, treated by TEVAR in the acute (1-14 days, $\mathrm{n}=130)$ or subacute (15-90 days, $\mathrm{n}=137)$ phase. No difference was observed in early outcomes between the groups. However, only those treated acutely suffered aortic rupture, RTAD, and disabling stroke. Interestingly, 30 -day mortality in the acute group was five times that of the subacute group (3.8\% vs. $0.7 \%)$ but not statistically significant $(\mathrm{P}=0.11)$, which suggests that intervention timing is not associated with 30-day mortality. Multivariable logistic analyses also suggested that the timing of the TEVAR did not independently correlate with 30-day outcomes. Finally, Xie et al. [37] were unable to reach a definitive consensus but indicated that TEVAR for high risk un-TBAD in the acute phase was associated with a propensity toward higher rates of early complications but comparable late outcomes.

Two studies analysed data from the SVS Vascular Quality Initiative (VQI) TEVAR for TBAD project. Wang et al. [38] reported similar findings to Xie et al. [37] as they could not find clearly defined patterns in mortality or re-intervention associated with timing of TEVAR. The authors stated that intervention during the acute phase of dissection rather than chronic showed a trend towards increased 30-day mortality and decreased freedom from reintervention. Torrent et al. [39] presented similar findings with fewer patients requiring re-intervention within 30 days and one year in the subacute phase relative to the acute phase $(\mathrm{P}=0.02$ and $\mathrm{P}=0.007$, respectively). Crude data prior to propensity matching highlighted an increased mortality within 30 days in the $1-14$ day group ( $7.5 \%$ compared to $2.7 \%$ in the $15-90$ day group, $\mathrm{P}=0.021$ ). However, multivariate analysis for long-term survival, complications, or long-term reintervention demonstrated no differences. Similar to the two aforementioned studies [37][38], Torrent and colleagues [39] concluded that the timing of intervention does not appear to be independently predictive of mortality or postoperative complications; however, considering whether to intervene in the acute phase is a decision that must be made with much caution.

Miyairi et al. [40] retrospectively investigated 680 patients in Japan over a 5 year time period who underwent TEVAR for TBAD. These were classified as hyperacute $(n=295$, performed within 24 hours of presentation of symptoms), acute ( $\mathrm{n}=97,24$ hours to 14 days) and subacute ( $\mathrm{n}=288,14$ days to 6 weeks). Primary end point was 30-day operative mortality. Outcomes for TEVAR were worse for hyperacute patients as these had more frequent immediate life-threatening complications such as rupture or malperfusion. Additionally, TEVAR performed acutely did not appear to increase the risk of added complications compared to subacute patients. To further verify this, hyperacute patients had a higher operative mortality and severe complications including aortic dissection compared to acute and subacute $(11.9 \%, 0 \%, 1.7 \% ; \mathrm{P}<0.001$; and $32.5 \%$, $10.3 \%, 8.3 \% ; \mathrm{P}<0.001$, respectively). Nonetheless, no differences were reported for these outcomes between acute and subacute phases $(\mathrm{P}=0.191$ and $\mathrm{P}=0.553$, respectively $)$.

Finally, literature reviews by Alfson et al. [2] and Matsuda et al. [41] supported TEVAR for un-TABD and discussed timing of intervention. Both concluded that intervention during the subacute phase of TBAD improved survival. Based on this, the subacute phase (14-90 days after symptom onset), appears to be the optimal TEVAR therapeutic window for un-TBAD. However, future randomized control trials and observational studies, investigating TEVAR for un-TBAD need to incorporate timing of intervention into their design to provide more definitive data to further optimise clinical practice and decision making. Also, closer scrutiny of the initial CT scans and clinical presentation may allow accurate initial classification of the TBAD as to whether they are complicated, uncomplicated or high risk uncomplicated. It may well be a significant number may be part of the latter group particularly as is often the case, the disease process is evolving and not clear cut to place into a specific category.

Conclusion 
TEVAR has proven to be a safe and effective treatment for un-TBAD in combination with OMT through comparable survival outcomes, improved aortic remodelling, and relatively low periprocedural added risks. Though the timing of intervention remains controversial, it is becoming clear that performing TEVAR during the subacute phase of un-TBAD yields better outcomes compared to earlier and delayed ( $>90$ days) intervention. Further research is required into both short and long-term outcomes of TEVAR in addition to its optimal therapeutic window for un-TBAD. With stronger evidence, TEVAR is likely to be adopted as the gold-standard intervention for un-TBAD with definitive timeframe guidelines.

\section{References}

1. Bossone E, LaBounty T, Eagle K. Acute aortic syndromes: diagnosis and management, an update. European Heart Journal. 2017;39(9):739-749d.

2. Alfson D, Ham S. Type B Aortic Dissections: Current Guidelines for Treatment. Cardiology Clinics. 2017;35(3):387-410.

3. Bedi V, Swain P, Yadav A. Medical therapy versus TEVAR for uncomplicated type B aortic dissection. Indian Journal of Thoracic and Cardiovascular Surgery. 2019;35(S2):174-178.

4. Criado FJ. Aortic dissection: a 250-year perspective. Tex Heart Inst J. 2011;38(6):694-700.

5. Zafar M, Li Y, Rizzo J, Charilaou P, Saeyeldin A, Velasquez C et al. Height alone, rather than body surface area, suffices for risk estimation in ascending aortic aneurysm. The Journal of Thoracic and Cardiovascular Surgery. 2018;155(5):1938-1950.

6. Nienaber C, Clough R. Management of acute aortic dissection. The Lancet. 2015;385(9970):800-811.

7. Riambau V et al. Editor's Choice - Management of Descending Thoracic Aorta Diseases: Clinical Practice Guidelines of the European Society for Vascular Surgery (ESVS). Eur J Vasc Endovasc Surg. 2017 Jan;53(1):4-52.

8. Daily P, Trueblood H, Stinson E, Wuerflein R, Shumway N. Management of Acute Aortic Dissections. The Annals of Thoracic Surgery. 1970;10(3):237-247.

9. Cooper M, Hicks C, Ratchford E, Salameh M, Malas M. Diagnosis and treatment of uncomplicated type B aortic dissection. Vascular Medicine. 2016;21(6):547-552.

10. Lombardi J, Hughes G, Appoo J, Bavaria J, Beck A, Cambria R et al. Society for Vascular Surgery (SVS) and Society of Thoracic Surgeons (STS) reporting standards for type B aortic dissections. Journal of Vascular Surgery. 2020;71(3):723-747

11. Malaisrie S, Mehta C. Updates on Indications for TEVAR in Type B Aortic Dissection. Innovations: Technology and Techniques in Cardiothoracic and Vascular Surgery. 2020;15(6):495-501.

12. Tadros R, Tang G, Barnes H, Mousavi I, Kovacic J, Faries P et al. Optimal Treatment of Uncomplicated Type B Aortic Dissection. Journal of the American College of Cardiology. 2019;74(11):1494-1504.

13. Howard C, Sheridan J, Picca L, Reza S, Smith T, Ponnapalli A et al. TEVAR for complicated and uncomplicated type B aortic dissection-Systematic review and meta-analysis. Journal of Cardiac Surgery. 2021;36(10):3820-3830.

14. Krol E, Panneton JM. Uncomplicated Acute Type B Aortic Dissection: Selection Guidelines for TEVAR. Ann Vasc Dis. 2017;10(3):165-169.

15. Elefteriades JA. Natural history of thoracic aortic aneurysms: indications for surgery, and surgical versus nonsurgical risks. Ann Thorac Surg. 2002;74(5):S1877-S1898.

16. Rozado J, Martin M, Pascual I, Hernandez-Vaquero D, Moris C. Comparing American, European and Asian practice guidelines for aortic diseases. J Thorac Dis. 2017;9(Suppl 6):S551-S560.

17. Trimarchi S, Jonker FH, Hutchison S, et al. Descending aortic diameter of $5.5 \mathrm{~cm}$ or greater is not an accurate predictor of acute type B aortic dissection. J Thorac Cardiovasc Surg. 2011;142(3):e101-e107.

18. Hossack M, Patel S, Gambardella I, Neequaye S, Antoniou G, Torella F. Endovascular vs. Medical Management for Uncomplicated Acute and Sub-acute Type B Aortic Dissection: A Meta-analysis. European Journal of Vascular and Endovascular Surgery. 2020;59(5):794-807.

19. Enezate T, Omran J, Al-Dadah A, White C, Patel M, Mahmud E et al. Thoracic endovascular repair versus medical management for acute uncomplicated type B aortic dissection. Catheterization and Cardiovascular Interventions. 2017;91(6):1138-1143. 
20. Tsai TT, Nienaber CA, Eagle KA. Acute aortic syndromes. Circulation. 2005; 112: 3802-13.

21. Suzuki T, Eagle KA, Bossone E, Ballotta A, Froehlich JB, Isselbacher EM. Medical management in type B aortic dissection. Ann Cardiothorac Surg. 2014;3(4):413-417.

22. Brunkwall J, Kasprzak P, Verhoeven E, Heijmen R, Taylor P, Alric P et al. Endovascular Repair of Acute Uncomplicated Aortic Type B Dissection Promotes Aortic Remodelling: 1 Year Results of the ADSORB Trial. European Journal of Vascular and Endovascular Surgery. 2014;48(3):285-291.

23. Lombardi J, Cambria R, Nienaber C, Chiesa R, Teebken O, Lee A et al. Prospective multicenter clinical trial (STABLE) on the endovascular treatment of complicated type B aortic dissection using a composite device design. Journal of Vascular Surgery. 2012;55(3):629-640.e2.

24. Lombardi J, Gleason T, Panneton J, Starnes B, Dake M, Haulon S et al. STABLE II clinical trial on endovascular treatment of acute, complicated type B aortic dissection with a composite device design. Journal of Vascular Surgery. 2020;71(4):1077-1087.e2.

25. Nienaber C, Rousseau H, Eggebrecht H, Kische S, Fattori R, Rehders T et al. Randomized Comparison of Strategies for Type B Aortic Dissection: the INvestigation of STEnt Grafts in Aortic Dissection (INSTEAD) trial. Circulation. 2009;120(25):2519-2528.

26. Nienaber C, Kische S, Rousseau H, Eggebrecht H, Rehders T, Kundt G et al. Endovascular Repair of Type B Aortic Dissection: Long-term Results of the Randomized Investigation of Stent Grafts in Aortic Dissection Trial. Circulation: Cardiovascular Interventions. 2013;6(4):407-416.

27. Brunkwall J, Lammer J, Verhoeven E, Taylor P. ADSORB: A Study on the Efficacy of Endovascular Grafting in Uncomplicated Acute Dissection of the Descending Aorta. Journal of Vascular Surgery. 2012;56(1):279.

28. Song, J.M.; Kim, S.D.; Kim, J.H.; Kim, M.-J.; Kang, D.H.; Seo, J.B.; Lim, T.-H.; Lee, J.W.; Song, M.G.; Song, J.-K. Long-term predictors of descending aorta aneurysmal change in patients with aortic dissection. J. Am. Coll. Cardiol. 2007, 50, 799-804.

29. Yuan X, Clough R, Nienaber C. Management of Uncomplicated Type B Aortic Dissection. Hearts. 2020;1(1):14-24.

30. Qin YL, Wang F, Li TX, Ding W, Deng G, Xie B, Teng GJ. Endovascular Repair Compared With Medical Management of Patients With Uncomplicated Type B Acute Aortic Dissection. J Am Coll Cardiol. 2016 Jun 21;67(24):2835-42.

31. Xiang D, Kan X, Liang H, Xiong B, Liang B, Wang L et al. Comparison of mid-term outcomes of endovascular repair and medical management in patients with acute uncomplicated type B aortic dissection. The Journal of Thoracic and Cardiovascular Surgery. 2021;162(1):26-36.e1.

32. Chen Y, Zhang S, Liu L, Lu Q, Zhang T, Jing Z. Retrograde Type A Aortic Dissection After Thoracic Endovascular Aortic Repair: A Systematic Review and Meta-Analysis. J Am Heart Assoc. 2017;6(9):e004649. Published 2017 Sep 22. doi:10.1161/JAHA.116.004649

33. Lee S, Kang W, Ko Y, Woo Y, Ahn C, Won J et al. Aortic Remodeling and Clinical Outcomes in Type B Aortic Dissection According to the Timing of Thoracic Endovascular Aortic Repair. Annals of Vascular Surgery. 2020;67:322-331.

34. Desai N, Gottret J, Szeto W, McCarthy F, Moeller P, Menon R et al. Impact of timing on major complications after thoracic endovascular aortic repair for acute type B aortic dissection. The Journal of Thoracic and Cardiovascular Surgery. 2015;149(2):S151-S156.

35. The VIRTUE Registry Investigators. The VIRTUE Registry of Type B Thoracic Dissections - Study Design and Early Results. Journal of Vascular Surgery. 2011;53(2):551.

36. The VIRTUE Registry Investigators. Mid-term Outcomes and Aortic Remodelling After Thoracic Endovascular Repair for Acute, Subacute, and Chronic Aortic Dissection: The VIRTUE Registry. Journal of Vascular Surgery. 2014;60(4):1097.

37. Xie E, Yang F, Liu Y, Xue L, Fan R, Xie N et al. Timing and Outcome of Endovascular Repair for Uncomplicated Type B Aortic Dissection. European Journal of Vascular and Endovascular Surgery. 2021;61(5):788-797.

38. Wang G, Cambria R, Lombardi J, Azizzadeh A, White R, Abel D et al. Thirty-day outcomes from the Society for Vascular Surgery Vascular Quality Initiative thoracic endovascular aortic repair for type B 
dissection project. Journal of Vascular Surgery. 2019;69(3):680-691.

39. Torrent D, Wang G, Malas M, Pearce B, Spangler E, Novak Z et al. Timing of Thoracic Endovascular Aortic Repair for Uncomplicated Acute Type B Aortic Dissection and Association With Complications. Journal of Vascular Surgery. 2018;67(6):e186.

40. Miyairi T, Miyata H, Chiba K, Nishimaki H, Ogawa Y, Motomura N et al. Influence of Timing After Thoracic Endovascular Aortic Repair for Acute Type B Aortic Dissection. The Annals of Thoracic Surgery. 2018;105(5):1392-1396.

41. Matsuda H. Treatment of uncomplicated type B aortic dissection. General Thoracic and Cardiovascular Surgery. 2016;65(2):74-79.
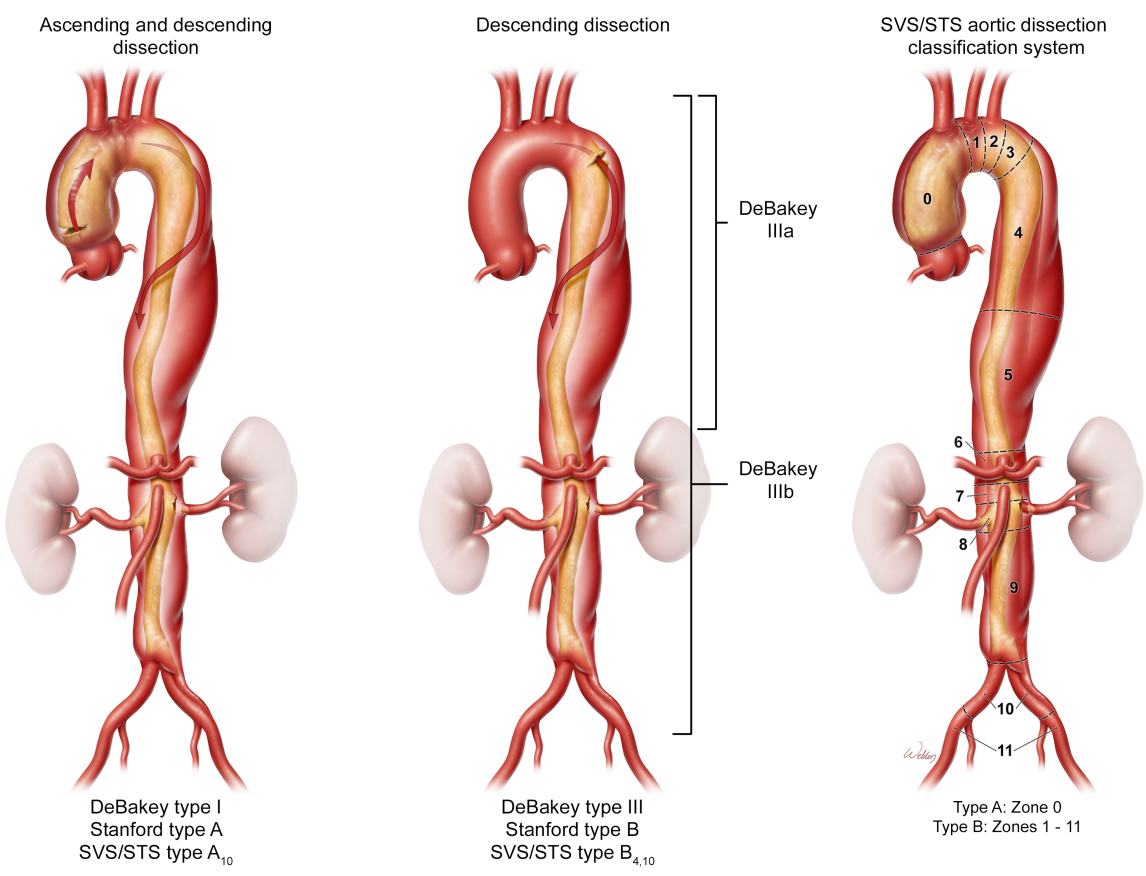


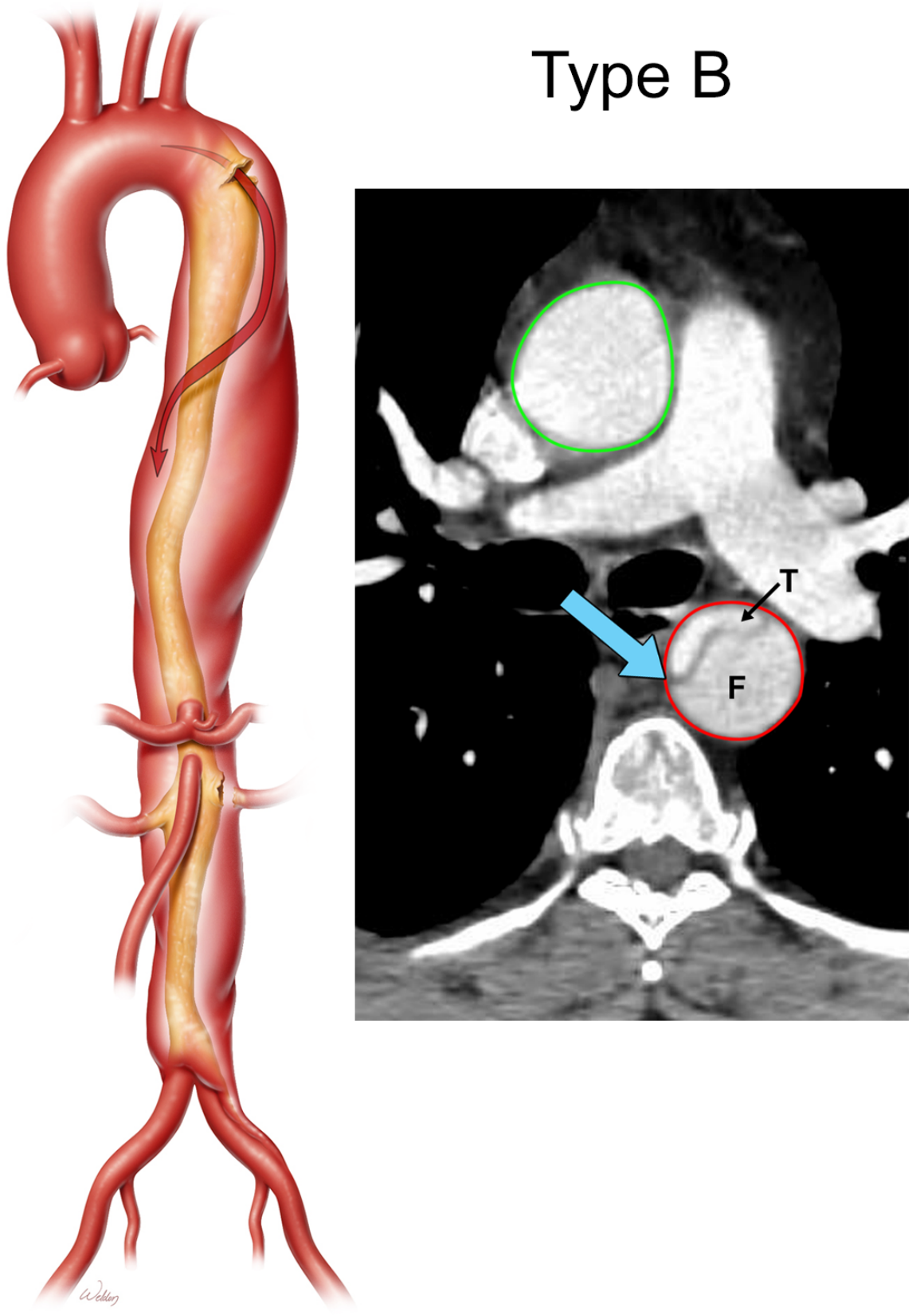




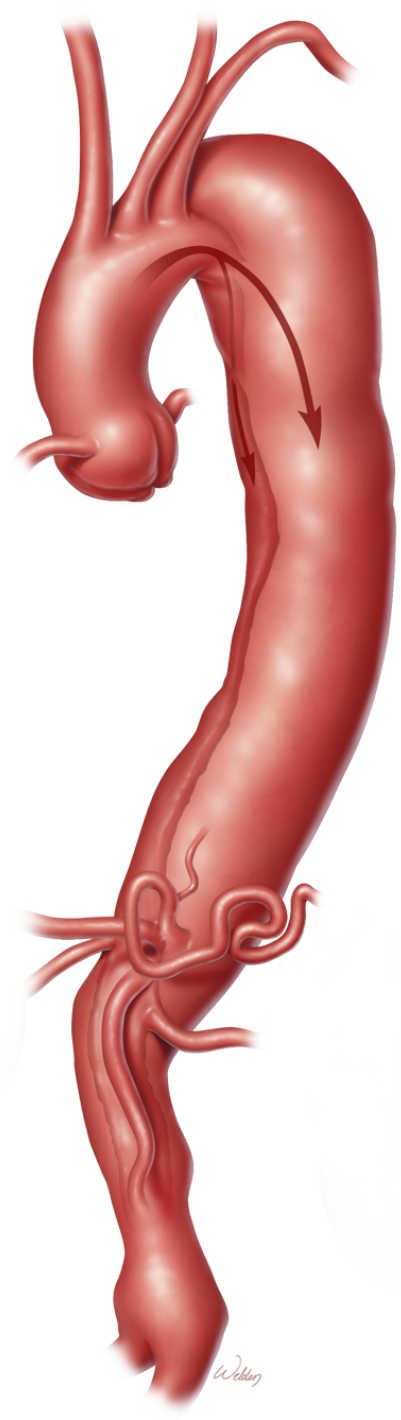

Type B

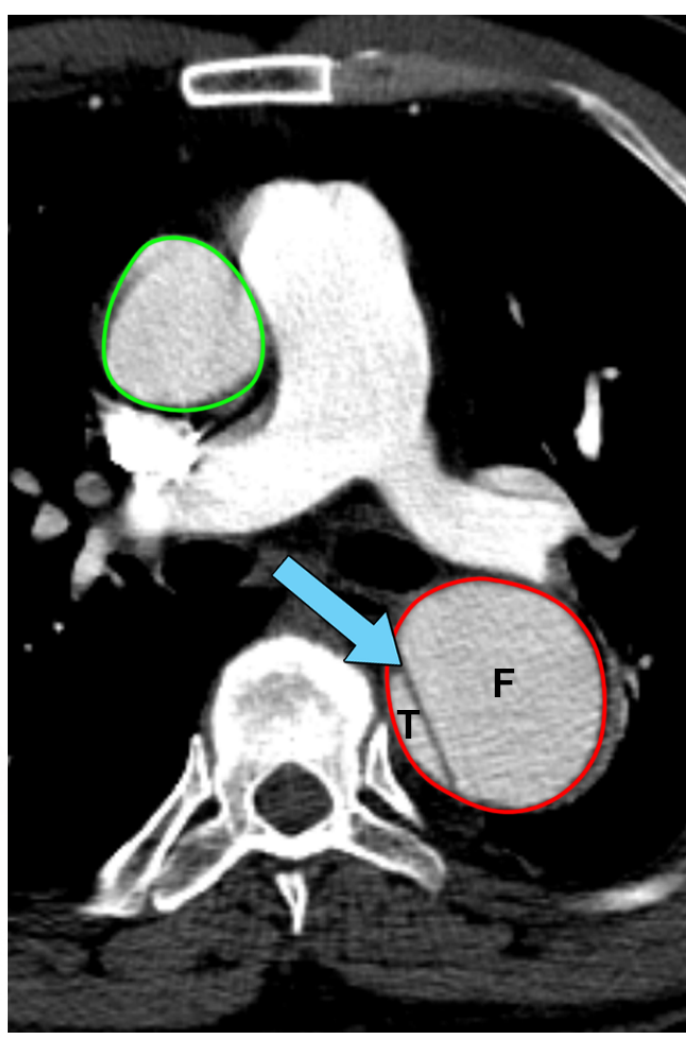

Hosted file

Tables (1\&amp;2).docx available at https://authorea.com/users/445492/articles/544996-whatis-the-optimal-timing-for-thoracic-endovascular-aortic-repair-in-uncomplicated-type-baortic-dissection-facts-fallacies-and-the-future 